\title{
El Laboratorio Virtual como herramienta didáctica en las enseñanzas de Ingeniería. Adaptación a la COVID-19
}

\begin{abstract}
D. E. Santiago-García ${ }^{a}$, B. Del Río-Gamerob y N. Melián-Martel ${ }^{\mathrm{c}}$
${ }^{a}$ Grupo de Innovación Educativa Interdisciplinar en Ingeniería Industrial (GIE3I), Departamento de Ingeniería de Procesos, Escuela de Ingenierías Industriales y Civiles, Universidad de Las Palmas de Gran Canaria, dsantiago@proyinves.ulpgc.es, ${ }^{b}$ Grupo de Innovación Educativa Interdisciplinar en Ingeniería Industrial (GIE3I), Departamento de Ingeniería de Procesos, Escuela de Ingenierías Industriales y Civiles, Universidad de Las Palmas de Gran Canaria beatriz.delrio@ulpgc.es y ${ }^{\mathrm{c}}$ Grupo de Innovación Educativa Interdisciplinar en Ingeniería Industrial (GIE3I), Departamento de Ingeniería de Procesos, Escuela de Ingenierías Industriales y Civiles, Universidad de Las Palmas de Gran Canaria noemi.melian@ulpgc.es
\end{abstract}

\section{\$EWWFW}

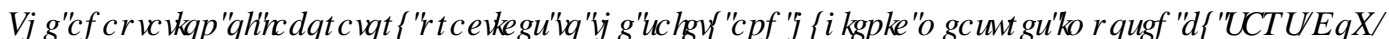

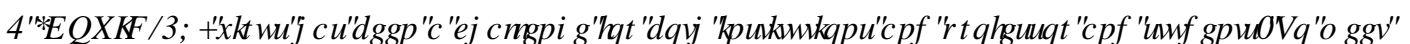

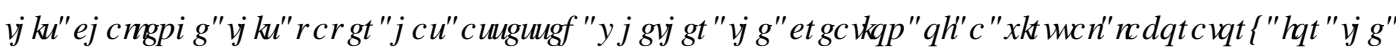

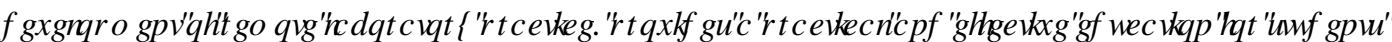

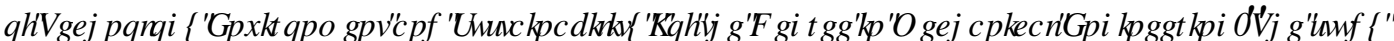

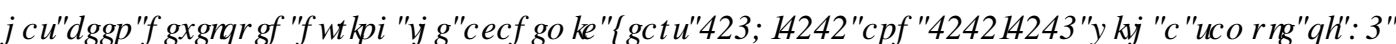

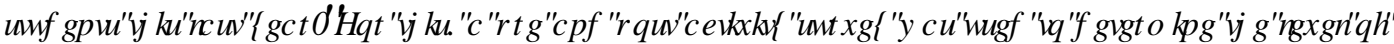

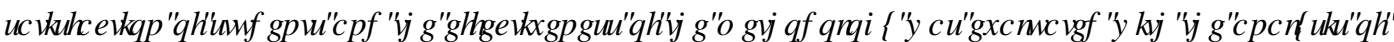

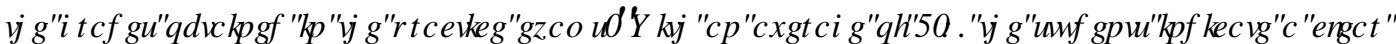

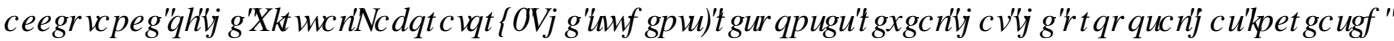

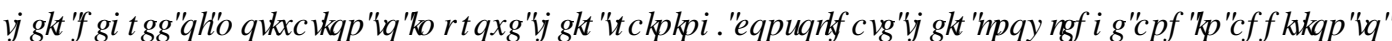

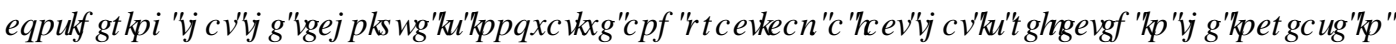
WKHDYHDJ HJU WOHRIIKKHHDP VRIISUFWFHप

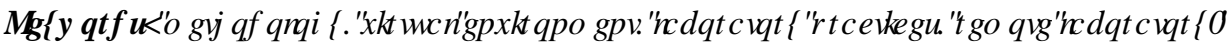

\section{HXP HQ}

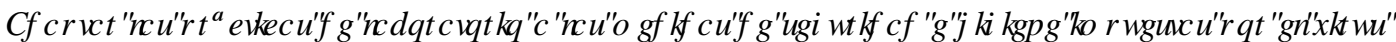

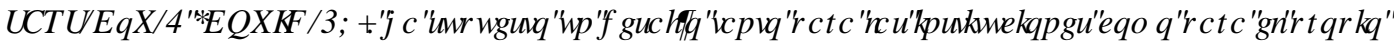

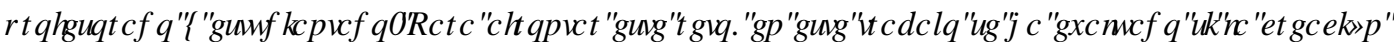

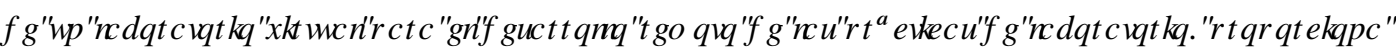

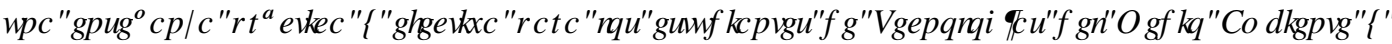

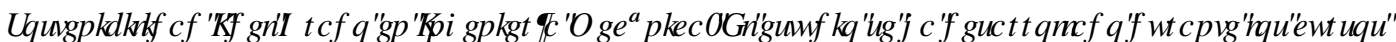

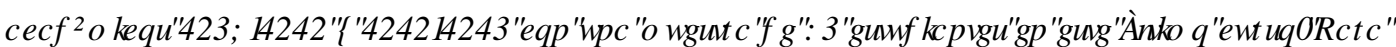

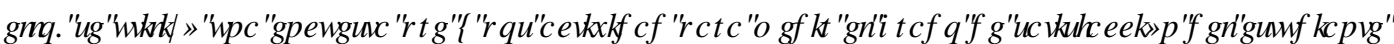

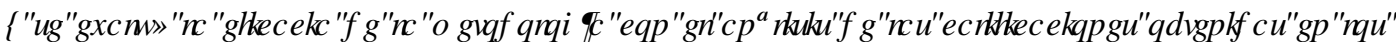

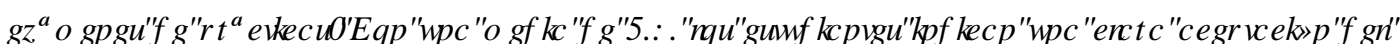

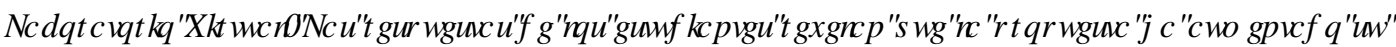

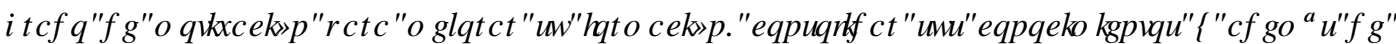

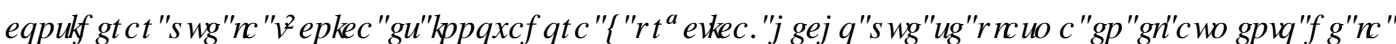
FDOIFDFIY QP HADCHIRVH i P HQHVGHSU FUFDVW

3 DOEUVFOYH

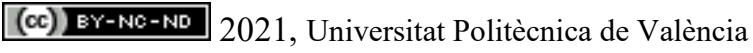

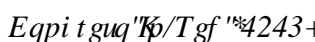




\section{Introducción}

Las circunstancias acontecidas desde el pasado año en relación a la pandemia generada por el virus SARSCoV-2 (COVID-19), ha traído consigo la necesidad de fomentar metodologías alternativas a la hora de afrontar la impartición de la docencia en las aulas universitarias. Durante este tránsito, son muchos los grados oficiales que se encuentran en la modalidad semi/no presencial, alcanzando un 9,2\% de la oferta total del territorio nacional (Ministerio de Ciencia Educación y Universidades, 2020). Dentro de ello, las titulaciones que disponen de prácticas de laboratorio en sus planes de estudio se han encontrado con una problemática importante a la hora de gestionar dichas prácticas debiendo cumplir en todo momento y de forma paralela con las medidas sanitarias de higiene y seguridad. La imposibilidad de asistir a los laboratorios, o la necesidad de disponer de suficientes equipos para que cada estudiante pueda llevar su práctica de forma individual en un espacio seguro (presencialidad adaptada), han sido algunos de los factores puestos sobre la mesa a la hora de discernir de qué manera se puede evitar la pérdida de este tipo de sesiones de formación. No se debe olvidar que los laboratorios son una piedra angular en titulaciones como las ingenierías, arquitectura o la rama de la salud, que sirve para evaluar y afianzar los conocimientos adquiridos en las sesiones teóricas. Además de ser un espacio donde las competencias generales y transversales como son el trabajo autónomo y en equipo o la promoción de la investigación entre los jóvenes, se desarrollan en un mayor grado, contribuyendo a la mejora continua de la profesión que posteriormente ejercerán.

Es por ello que este trabajo plantea una nueva versión de estas clases prácticas que pueden fortalecer y/o en algún caso excepcional, suplantar el estilo de práctica presencial, con la intención de que el estudiantado complemente y/o adquiera de alguna manera las habilidades y competencias pertinentes.

Esta investigación pretende introducir en la asignatura Tecnologías del Medio Ambiente y Sostenibilidad I del Grado en Ingeniería Mecánica, impartida en la Escuela de Ingenieros Industriales y Civiles de la Universidad de Las Palmas de Gran Canaria, un entorno virtual donde poder disfrutar de un laboratorio remoto, que sirva como herramienta útil de enseñanza/aprendizaje activa y complementaria a la tradicional clase magistral (Garai y Saratxaga, 2018). Con la idea de conseguir una similitud a las prácticas presenciales, se mantiene el modelo de laboratorio formal descrito por Kirschner (Kirschner, 1992), el cual sigue liderando las metodologías aplicadas en los laboratorios actuales (Flores et al., 2009; Sebastia, 1987). Para ello, se puede hacer uso de simulaciones, videos producidos y editados por el profesorado o incluso videoconferencias síncronas donde el/la estudiante puede ver el desarrollo del ensayo/experimentación en tiempo real (De La Torre et al., 2013; Vary, 2000).

Si bien es cierto que existen bastantes referencias del uso de laboratorios virtuales en cualquier área de conocimiento (Pérez, 2020; Universidad Politécnica de Madrid, 2020; Universidad Europea, 2020; Universitat de Valencia, 2020; Universitat Internacional de Catalunya, 2020), liderado en todo momento por el sector de las telecomunicaciones e informática (Maurel et al., 2014; Castillo y Guerrero, 2016; Infante, 2014; Zuluaga, 2010), lo más cercano al ámbito de la ingeniería ambiental serían los laboratorios de la titulación de química (Gobierno de Canarias, 2020; GRAO, 2020).

Sin embargo, las prácticas realizadas en la titulación de química suelen englobar el desarrollo de un solo concepto, mientras que las asignaturas enmarcadas dentro de la ingeniería ambiental disponen de prácticas que se caracterizan por la necesidad de dar a conocer al estudiante un conglomerado de conceptos que debe de conocer y tratar de forma simultánea para comprender el objetivo final de la temática abordada. A modo de ejemplo, en las prácticas referentes a los tratamientos de aguas residuales y/o de abastacimiento para consumo humano, el estudiantado debe de abarcar diferentes conceptos que van desde los diversos parámetros de medida hasta la legislación a aplicar para confirmar la validez de dichos ensayos. 
Todo esto hace que el laboratorio remoto sirva como apoyo para que el trabajo físico en el laboratorio real, donde el estudiantado deberá de enfrentarse no sólo a uno, sino a dos, tres o más puestos, sea mucho más fácil y eficiente en la adiquisición de conocimiento ya que tendrán acceso y podrán visualizar el número de veces que estimen oportuno todo el contenido generado y colgado en el entorno moodle.

La selección de cada una de las posibles técnicas (vídeos, ensayos online, etc) radica en el tipo de práctica, material necesario, porcentaje de tiempo dedicado a la introducción teórica y explicación de la práctica, distancias de seguridad necesarias entre los puestos de trabajo, los diferentes equipos empleados y el grado de complejidad de la práctica en cuanto a tiempo necesario para poder desarrollarla en el horario establecido por una sola persona y no por parejas o equipos. La decisión tomada también se debe en gran medida a la disminución de costes que la Universidad hubiera debido de afrontar debido al desdoble de los grupos en el caso de que la práctica se focalizara en la explicación y empleo de un único equipo.

Con el objetivo de evaluar la mejora en la consolidación del conocimiento de los estudiantes, se emplearon cuestionarios virtuales alojados en el Moodle de la asignatura, que los estudiantes debían de cumplimentar antes y después de cada práctica (mismo cuestionario antes y después de cada práctica). Dichos cuestionarios permiten cuantificar los conocimientos adquiridos, y analizar si el laboratorio virtual puede ser una herramienta de apoyo en los años venideros, reforzando el aprendizaje de una forma didáctica (Tomasi, 2018).

\section{Objetivos}

El objetivo de este trabajo fue adaptar las prácticas de laboratorio a las medidas de seguridad e higiene impuestas por la pandemia debida al virus COVID-19. Para ello se creó un laboratorio virtual y se evaluó tanto la opinión de los estudiantes sobre esta metodología como sus resultados académicos. Para la consecusión de este objetivo general se establecieron los siguientes objetivos específicos:

- Conocer la opinión de los estudiantes sobre esta metodología al comienzo del curso (pre actividad), en cuanto a sus expectativas/motivación, utilidad, contenido/método y herramientas.

- Conocer la opinión de los estudiantes una vez empleada la metodología (pos actividad) para evaluar su grado de satisfacción con la actividad.

- Evaluar el empleo de esta metodología sobre el resultado académico de los estudiantes, comparando la calificación obtenida por los alumnos de este curso con los resultados de compañeros que cursaron las mismas prácticas de forma $100 \%$ presencial en cursos anteriores.

\section{Desarrollo de la innovación}

En este trabajo se desarrolló un laboratorio virtual con vídeos donde se detalló el procedimiento experimental a seguir en cada una de las prácticas. Además, el laboratorio virtual cuenta con los tradicionales guiones de práctica donde el alumnado puede consultar los conceptos a desarrollar en las prácticas, así como el procedimiento experimental escrito.

Esta experiencia se llevó a cabo en la asignatura de Tecnologías del Medio Ambiente y Sostenibilidad I del segundo curso del Grado en Ingeniería Mecánica, en la Universidad de Las Palmas de Gran Canaria. El número total de estudiantes matriculados fue de 81 , de los cuales 18 no realizaron las prácticas al ser repetidores y haberlas superado en el curso anterior. En la asignatura se incluyeron tres prácticas de laboratorio. En el curso 2020/2021 se realizaron las prácticas 1 y 3 de forma telepresencial, empleando únicamente el laboratorio virtual y una sesión de una hora a través de la plataforma Teams para explicar a

(c)) BY-NC-ND 2021, Universitat Politècnica de València

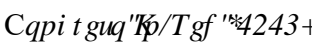


los alumnos el contenido del mismo. Para la práctica 2 se solicitó a los alumnos que visualizaran el laboratorio virtual en casa antes de acudir al laboratorio y posteriormente acudieron al laboratorio para la realización de la misma. La evaluación de las prácticas se realizó mediante exámenes tipo test.

Para evaluar la opinión de los estudiantes acerca de esta experiencia se empleó el cuestionario denominado ( [SHFWWDV, desarrollado por Albert et DO (Albert Gómez et al., 2016). Se pidió a los estudiantes que respondieran al cuestionario de forma anónima y voluntaria, antes de comenzar las prácticas de laboratorio y también después de haberlas completado. El cuestionario consistió en 12 preguntas con las que se midieron los siguientes indicadores: expectativas/motivación, utilidad, contenido/método utilizado, y herramientas. Las preguntas siguieron una escala tipo Likert del 1 al 5, siendo 1 IRLDP HQWAHQGHMFXHGR, 2 HQGHDFXHGR, 3 QLGHDFXHGRIQLHQGHDFXHGR, 4 GHDFXHGR y 5 URLDP HQWGHDFXHR.

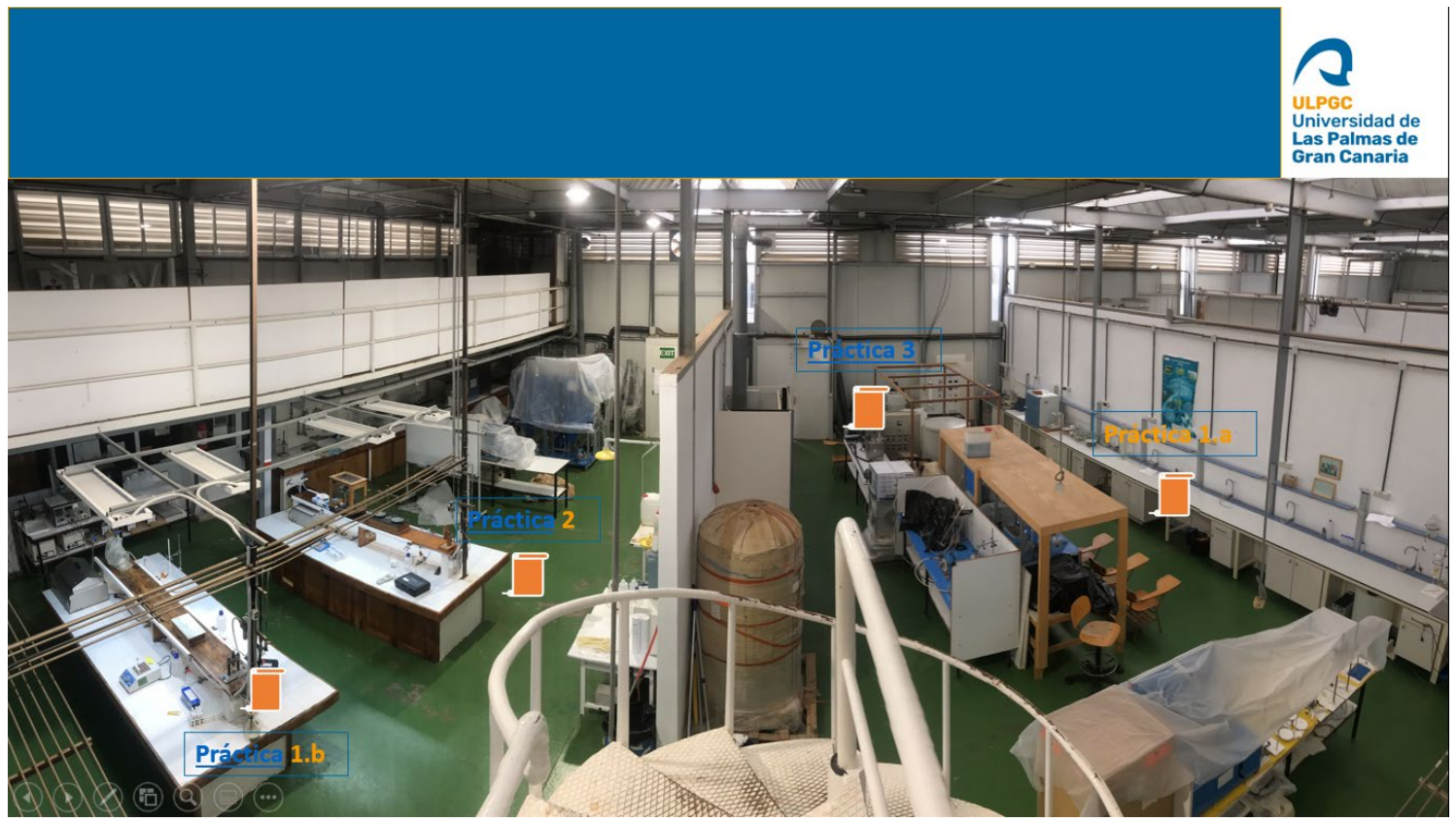

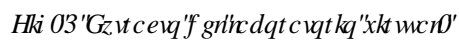

El detalle de las preguntas del cuestionario empleado puede consultarse en la Tabla 1.

7DECDIप( QFXHWDSHSRVDFWHGDG

\begin{tabular}{|c|c|c|}
\hline $\mathbf{N}^{\mathbf{o}}$ & Indicador & Ítem \\
\hline 1 & \multirow{3}{*}{ Expectativas/Motivación } & $\begin{array}{l}\text { A través de estas prácticas espero obtener/he obtenido nuevas habilidades y } \\
\text { conocimientos para desempeñar de forma satisfactoria mi labor profesional }\end{array}$ \\
\hline 2 & & $\begin{array}{l}\text { La realización de estas prácticas aumentará/ha aumentado mis conocimientos para } \\
\text { adecuarme a las demandas del mundo laboral }\end{array}$ \\
\hline 3 & & $\begin{array}{l}\text { Mi motivación respecto a estas prácticas se basa/ha basado en el interés por mejorar mi } \\
\text { formación }\end{array}$ \\
\hline 4 & \multirow{3}{*}{ Utilidad } & $\begin{array}{l}\text { Considero muy útil la realización de estas prácticas porque mejoran/han mejorado las } \\
\text { competencias profesionales que se requieren en la sociedad laboral actual }\end{array}$ \\
\hline 5 & & Indique el grado de utilidad de las prácticas para su desarrollo personal y profesional \\
\hline 6 & & $\begin{array}{l}\text { Espero a través de estas prácticas estar/después de realizar estas prácticas estoy } \\
\text { plenamente actualizado en las nuevas tendencias dentro de este ámbito laboral y } \\
\text { profesional. }\end{array}$ \\
\hline 7 & \multirow{2}{*}{ Contenido/Método } & $\begin{array}{l}\text { Creo que el contenido de estas prácticas consolidará/ha consolidado mis conocimientos } \\
\text { básicos y ampliará/ha ampliado el uso de las herramientas en ellas utilizadas }\end{array}$ \\
\hline 8 & & $\begin{array}{l}\text { Considero necesario que/considero que los contenidos didácticos de las prácticas } \\
\text { sean/son precisos, comprensibles y estén actualizados }\end{array}$ \\
\hline
\end{tabular}

(cc) BY-NC-ND 2021, Universitat Politècnica de València

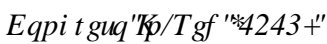




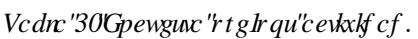

\begin{tabular}{|c|c|c|}
\hline 9 & & $\begin{array}{l}\text { La realización de las prácticas a distancia con el laboratorio virtual es una } \\
\text { metodología innovadora y muy práctica }\end{array}$ \\
\hline 10 & \multirow{3}{*}{ Herramientas } & $\begin{array}{l}\text { Espero que los recursos incluidos en estas prácticas me ayuden/los recursos incluidos } \\
\text { en estas prácticas me han ayudado a relacionar la parte práctica con la teoría, de forma } \\
\text { que resulte/resulta más fácil la asimilación de contenidos }\end{array}$ \\
\hline 11 & & Creo que las herramientas informáticas utilizadas son las más adecuadas para su fin \\
\hline 12 & & $\begin{array}{l}\text { Debe existir/Existe una adecuación real y efectiva entre los recursos multimedia que se } \\
\text { ponen a disposición en la plataforma y sus usos didácticos }\end{array}$ \\
\hline
\end{tabular}

Por último, para evaluar la eficacia de la metodología frente a las prácticas de laboratorio presenciales, se analizó lo siguiente:

- Las calificaciones obtenidas en los exámenes de prácticas por los alumnos que usaron el laboratorio virtual (curso 2020/2021) frente a quienes no lo hicieron (curso anterior).

- Las calificaciones de un mismo grupo de alumnos (curso 2020/2021) al realizar prácticas totalmente telepresenciales (prácticas 1 y 3 ) frente a la realización de otra práctica presencial apoyada por el contenido del laboratorio virtual (práctica 2).

\section{Resultados}

A continuación, se detallan los resultados obtenidos en este trabajo. Comenzaremos con los resultados de la opinión de los estudiantes acerca del laboratorio virtual: encuesta pre- y pos actividad. A continuación, se presentará el resultado del análisis de las calificaciones de los alumnos tras la actividad.

\subsection{La opinión de los estudiantes}

Como se comentó anteriormente, los estudiantes respondieron a una misma encuesta antes y después de haber cursado las prácticas con el laboratorio virtual.

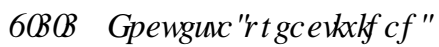

A continuación se muestran los resultados de la encuesta preactividad. Esta encuesta fue completada por 21 alumnos (el 33\% del total).

Se observa que la mayoría de los alumnos ha estado de acuerdo o totalmente de acuerdo con los ítems 1 a 6 , relacionados con las expectativas/motivación y utilidad. En este sentido esperaban que las prácticas aumentaran sus habilidades de forma que les facilitara la adaptación al mundo laboral y se sentirán motivados por mejorar su formación.

En cuanto al contenido/método, los alumnos coincidieron en que los contenidos de las prácticas deben estar actualizados y ser precisos y comprensibles; y la mayoría creyó que el desarrollo de las prácticas consolidaría sus conocimientos. Sin embargo, no estuvieron, por lo general, ni de acuerdo ni en desacuerdo con que la metodología del laboratorio virtual fuera innovadora.

Del mismo modo, la mayoría estuvo de acuerdo o totalmente de acuerdo en que las herramientas informáticas empleadas deben facilitar la relación entre la parte práctica y la teoría. 


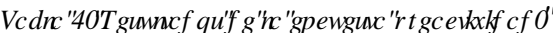

\begin{tabular}{ccccccc}
\hline \multirow{2}{*}{ Pregunta } & \multicolumn{5}{c}{ Respuestas (\%) } & Media \\
\cline { 2 - 5 } & $\mathbf{1}$ & $\mathbf{2}$ & $\mathbf{3}$ & $\mathbf{4}$ & $\mathbf{5}$ & $\mathbf{4 , 0 5}$ \\
\hline $\mathbf{1}$ & $4,76 \%$ & $4,76 \%$ & $14,29 \%$ & $33,33 \%$ & $42,86 \%$ & $\mathbf{3 , 7 6}$ \\
$\mathbf{2}$ & $9,52 \%$ & $0,00 \%$ & $28,57 \%$ & $28,57 \%$ & $33,33 \%$ & $\mathbf{4 , 1 4}$ \\
$\mathbf{3}$ & $4,76 \%$ & $4,76 \%$ & $9,52 \%$ & $33,33 \%$ & $47,62 \%$ & $\mathbf{3 , 9 0}$ \\
$\mathbf{4}$ & $4,76 \%$ & $4,76 \%$ & $19,05 \%$ & $38,10 \%$ & $33,33 \%$ & $\mathbf{3 , 8 5}$ \\
$\mathbf{5}$ & $4,76 \%$ & $9,52 \%$ & $9,52 \%$ & $42,86 \%$ & $28,57 \%$ & $\mathbf{3 , 6 7}$ \\
$\mathbf{6}$ & $4,76 \%$ & $4,76 \%$ & $28,57 \%$ & $42,86 \%$ & $19,05 \%$ & $\mathbf{3 , 7 6}$ \\
$\mathbf{7}$ & $4,76 \%$ & $4,76 \%$ & $23,81 \%$ & $42,86 \%$ & $23,81 \%$ & $\mathbf{4 , 4 8}$ \\
$\mathbf{8}$ & $4,76 \%$ & $0,00 \%$ & $9,52 \%$ & $14,29 \%$ & $71,43 \%$ & $\mathbf{3 , 3 8}$ \\
$\mathbf{9}$ & $14,29 \%$ & $4,76 \%$ & $38,10 \%$ & $14,29 \%$ & $28,57 \%$ & $\mathbf{4 , 2 4}$ \\
$\mathbf{1 0}$ & $0,00 \%$ & $4,76 \%$ & $14,29 \%$ & $33,33 \%$ & $47,62 \%$ & $\mathbf{3 , 8 1}$ \\
$\mathbf{1 1}$ & $4,76 \%$ & $4,76 \%$ & $28,57 \%$ & $28,57 \%$ & $33,33 \%$ & $\mathbf{4 , 1 4}$ \\
$\mathbf{1 2}$ & $0,00 \%$ & $4,76 \%$ & $14,29 \%$ & $42,86 \%$ & $38,10 \%$ & \\
\hline
\end{tabular}

\section{पMU ( QFXHMDSRLFWIGDO}

La encuesta posactividad la completaron 15 alumnos (el 24\%). Los resultados se muestran en la Tabla 3.

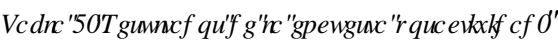

\begin{tabular}{ccccccc}
\hline \multirow{2}{*}{ Pregunta } & \multicolumn{7}{c}{ Respuestas (\%) } \\
\cline { 2 - 7 } & $\mathbf{1}$ & $\mathbf{2}$ & $\mathbf{3}$ & $\mathbf{4}$ & $\mathbf{5}$ & Media \\
\hline $\mathbf{1}$ & $0,00 \%$ & $6,67 \%$ & $40,00 \%$ & $46,67 \%$ & $6,67 \%$ & $\mathbf{3 , 5 3}$ \\
$\mathbf{2}$ & $0,00 \%$ & $13,33 \%$ & $13,33 \%$ & $60,00 \%$ & $13,33 \%$ & $\mathbf{3 , 7 3}$ \\
$\mathbf{3}$ & $0,00 \%$ & $0,00 \%$ & $20,00 \%$ & $40,00 \%$ & $40,00 \%$ & $\mathbf{4 , 2 0}$ \\
$\mathbf{4}$ & $0,00 \%$ & $13,33 \%$ & $26,67 \%$ & $40,00 \%$ & $20,00 \%$ & $\mathbf{3 , 6 7}$ \\
$\mathbf{5}$ & $0,00 \%$ & $6,67 \%$ & $26,67 \%$ & $60,00 \%$ & $6,67 \%$ & $\mathbf{3 , 6 7}$ \\
$\mathbf{6}$ & $0,00 \%$ & $20,00 \%$ & $40,00 \%$ & $33,33 \%$ & $6,67 \%$ & $\mathbf{3 , 2 7}$ \\
$\mathbf{7}$ & $0,00 \%$ & $0,00 \%$ & $20,00 \%$ & $53,33 \%$ & $26,67 \%$ & $\mathbf{4 , 0 7}$ \\
$\mathbf{8}$ & $0,00 \%$ & $0,00 \%$ & $0,00 \%$ & $60,00 \%$ & $40,00 \%$ & $\mathbf{4 , 4 0}$ \\
$\mathbf{9}$ & $6,67 \%$ & $0,00 \%$ & $40,00 \%$ & $13,33 \%$ & $40,00 \%$ & $\mathbf{3 , 8 0}$ \\
$\mathbf{1 0}$ & $0,00 \%$ & $6,67 \%$ & $6,67 \%$ & $73,33 \%$ & $13,33 \%$ & $\mathbf{3 , 9 3}$ \\
$\mathbf{1 1}$ & $0,00 \%$ & $6,67 \%$ & $46,67 \%$ & $13,33 \%$ & $33,33 \%$ & $\mathbf{3 , 7 3}$ \\
$\mathbf{1 2}$ & $0,00 \%$ & $6,67 \%$ & $26,67 \%$ & $26,67 \%$ & $40,00 \%$ & $\mathbf{4 , 0 0}$ \\
\hline
\end{tabular}

Tras el curso, la puntuación media obtenida en el cuestionario para cada uno de los ítems fue similar o ligeramente inferior para todos ellos excepto para las preguntas 3, 7 y 9. El aumento en la valoración es especialmente notable para los ítems 7 y 9, aumentando la media en 0,31 y 0,42 puntos, respectivamente. Esto indica que los alumnos se sintieron motivados para mejorar su formación, consolidaron sus conocimientos con el uso del laboratorio virtual y además consideraron que la técnica sí es innovadora y práctica.

En cuanto a los ítems que recibieron una peor valoración en el cuestionario posactividad respecto al cuestionario preactividad, los más destacables son el 1, 4 y 10, con una disminución media de 0,52, 023 y 
0,31 puntos, respectivamente. Los ítems 1 y 4 están relacionados con el enfoque de las prácticas de cara a la inserción laboral del alumnado. Cabe destacar que las prácticas de la asignatura están relacionadas con la caracterización de aguas residuales (práctica 1), aguas potables (práctica 2) y la contaminación ambiental en fuentes estacionarias (práctica 3). Los alumnos que participaron en el estudio cursan el Grado en Ingeniería Mecánica y los conceptos abarcados en las prácticas de esta asignatura no coinciden firmemente con sus expectativas laborales. El ítem 10 evalúa la relación entre las partes teórica y práctica de la asignatura; en futuras ediciones se profundizará en mejorar este aspecto.

La puntuación media fue superior a 3,6 en todos los casos y superior a 4 para cuatro ítems, lo cual indica una buena acogida del laboratorio virtual por parte de los alumnos. Estos resultados coinciden con los publicados por otros autores que han desarrollado trabajos similares (Albert Gómez et al., 2016).

\section{पMU \&DOIFDFIRQHMHQH i P HQHWGHSU FWFDV}

En la Fig. 2 se representa la nota media y la de los exámenes de las prácticas 1, 2 y 3 que obtuvieron los alumnos en el curso 2020/2021 (uso de laboratorio virtual) frente a las calificaciones que obtuvieron los alumnos en el curso anterior, 2019/2020 (prácticas presenciales).
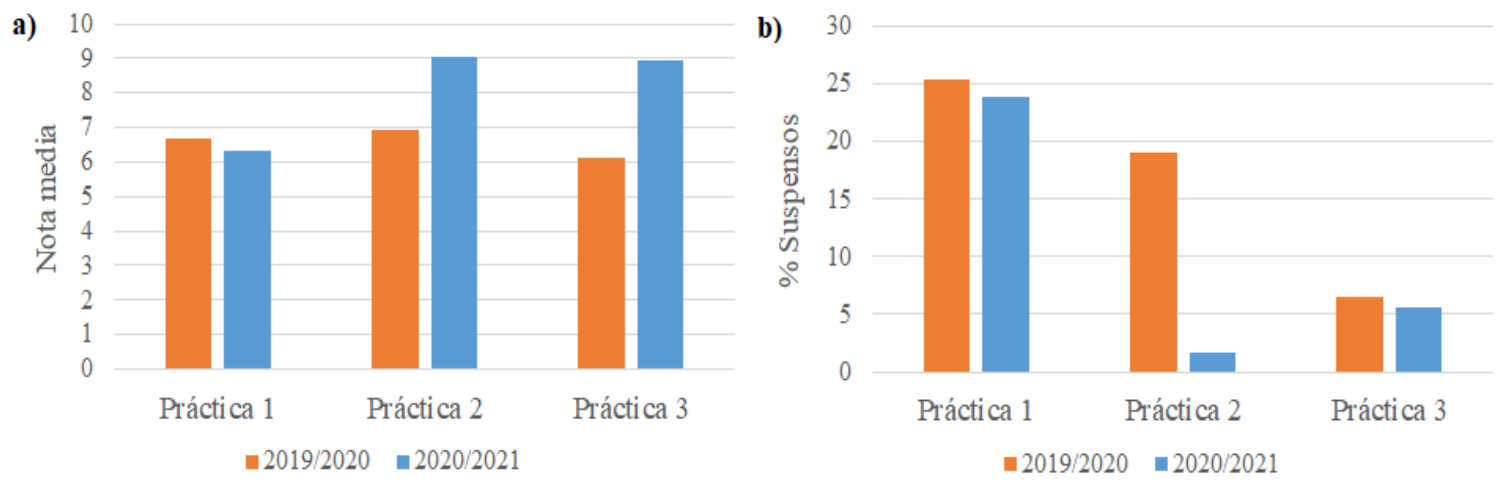

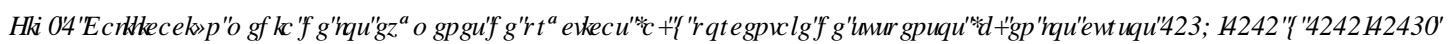

Se observa un claro aumento de la calificación media obtenida por los alumnos en los exámenes de las prácticas 2 y 3 si comparamos el curso 2020/2021 (laboratorio virtual) con el curso anterior (prácticas presenciales sin laboratorio virtual). Estos resultados coinciden con los de otros autores que no vieron una disminución en el aprendizaje final del alumnado por desarrollar las prácticas de forma no presencial (Long et al., 2012; Vincent-Lancrin, 2013).

Además, el porcentaje de suspensos disminuyó considerablemente en la evaluación de la práctica 2. Esta fue la práctica que se hizo en el curso 2020/2021 de forma presencial con el apoyo del laboratorio virtual.

Por otra parte, 63, 58 y 53 alumnos cursaron las prácticas 1, 2 y 3, respectivamente en el curso 2020/2021. En el curso 2019/2020 el número de alumnos que cursaron las prácticas 1, 2 y 3 fue de 55, 42 y 46, respectivamente. Se observa en este sentido un abandono de la asignatura con el transcurso del curso, siendo el porcentaje de abandono del 16\% en ambos cursos.

\section{Conclusiones}

En este trabajo se ha evaluado la adaptación de la docencia práctica presencial a la no presencialidad mediante la creación de un Laboratorio Virtual. 
El Laboratorio Virtual desarrollado para la asignatura de Tecnologías del Medio Ambiente y Sostenibilidad I en el Grado de Ingeniería Mecánica, se ha presentado como una alternativa para fortalecer los conocimientos técnicos y/o en casos requeridos suplantar a las tradicionales prácticas de laboratorio.

A partir de los resultados obtenidos de las encuestas pre y pos actividad se pone de manifiesto una clara aceptación y acogida del Laboratorio Virtual por parte de los estudiantes. Se observa que la valoración de los alumnos disminuye tras realizar la actividad, para los ítems relacionados con las expectativas/motivación $(-0,16)$, utilidad $(-0,27)$, herramientas $(-0,17)$, excepto en los ítems relacionados con el contenido/método $(+0,02)$.

En cuanto a las expectativas/motivación, la disminución más destacada la encontramos en las expectativas de los alumnos sobre la obtención de nuevas habilidades para el desempeño profesional, acentuándose esta percepción nuevamente en la siguiente categoría de preguntas (utilidad).

Por el contrario, los alumnos percibieron tras realizar la actividad que la asimilación de los conceptos básicos se ha visto favorecida empleando esta nueva metodología (categoría de preguntas contenido/método).

En cuanto a la opinión de los estudiantes sobre las herramientas empleadas, de cara a una perspectiva futura deberán emplearse herramientas multimedia más atractivas, ya que la valoración de los alumnos pos actividad nos indica que la herramienta empleada no cubrió sus expectativas. En este sentido, el Laboratorio Virtual fue realizado en power point con la ayuda de vídeos. En próximas ediciones se evaluará la opción de emplear software para realidad virtual.

Finalmente, el aumento de la calificación media de las notas de exámenes de prácticas confirma el aumento el aprendizaje final de los estudiantes. Además, en todas las prácticas se observó un descenso del número de estudiantes suspensos.

Aunque la propuesta no suple a los laboratorios presenciales en este campo, donde se hace necesario que el estudiante experimente de forma autónoma e interactúe de forma directa con el profesor, de los resultados de este trabajo se puede concluir que el Laboratorio Virtual es una herramienta de apoyo adecuada que además puede favorecer la asimilación de conceptos por parte de los estudiantes.

\section{Referencias}

ALBERT GÓMEZ, M.J., GARCÍA PÉREZ CALABUIG, M., DÍAZ ORUETA, G. (2016). "La evaluación como instrumento de formación para el aprendizaje a través de los laboratorios remotos". 5 HW' RFHPID8 QIY. REDUWVol. 14, p. 377.

CASTILLO, E., GUERRERO, C. (2016). "Implementación de prácticas de laboratorio en la educación virtual de los

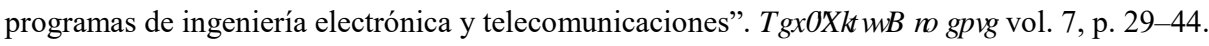

DE LA TORRE, L., HERADIO, R., JARA, C.A., SANCHEZ, J., DORMIDO, S., TORRES, F., CANDELAS, F.A. (2013). "Providing collaborative support to virtual and remote laboratories". , ( ( $\square$ TWQVI/ HDQW7HFKQRO vol. 6, p. $312-323$.

FLORES, J., CABALLERO SAHELICES, M.C., MOREIRA, M.A. (2009). "El laboratorio en la enseñanza de las ciencias: Una visión integral en este complejo ambiente de aprendizaje". 5HШ, QYHM,vol. 33, p. 75-111.

GARAI, G. G., y SARATXAGA, G. H. (2018) "Visita a empresa: una herramienta educativa para la universidad y una

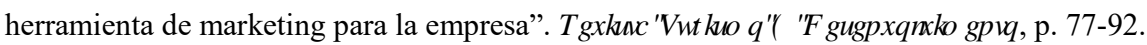

GOBIERNO DE CANARIAS. (2020). 3XECFDFIRQHD HMXHMDDV $<$ http://www3.gobiernodecanarias.org/medusa/ecoescuela/recursosdigitales/tag/laboratorio-virtual/> [consulta 15.02.2021]. 
GRAO. (2020). / DERWWRURV YLWDOAD GH TXtPIFD <https://www.grao.com/es/producto/laboratorios-virtuales-dequimica $>$ [consulta 15.02.2021].

KIRSCHNER, P.A. (1992). "Epistemology, practical work and Academic skills in science education". 6FHQFH ( GXFDURQ vol. 1, p. 273-299.

LONG, J.M., STANNARD, W.B., CHENERY, K., JOORDENS, M.A. (2012). "Physics practicals for distance

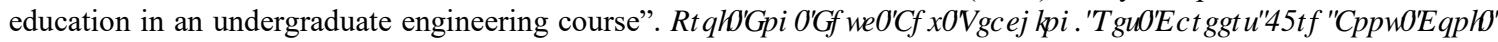
\$XWDDV. Assoc. Eng. Educ. p. 691-701.

MAUREL, M. C., DALFARO, N. A., SORIA, H. F. (2014). "El laboratorio virtual: Una herramienta para afrontar el

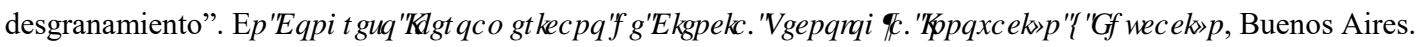

MINISTERIO DE CIENCIA EDUCACION Y UNIVERSIDADES. (2020). QEDU - Qué Estudiar y Dónde en la Universidad < https://www.educacion.gob.es/notasdecorte/compBdDo> [consulta 9.02.21].

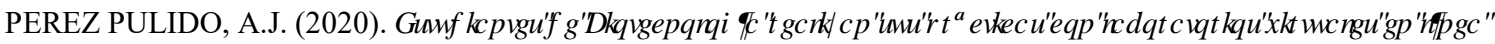
DUWVPVGHO\$ X(D) 9 IWDDOUniversidad Pablo Olavide. <https://www.upo.es/diario/comunidad/2020/03/estudiantes-debiotecnologia-realizan-sus-practicas-con-laboratorios-virtuales-en-linea-a-traves-del-aula-virtual/> [consulta 15.02.2021].

SEBASTIA, J.M. (1987). “QQué se pretende en los laboratorios de física universitaria? ” ( QMHDQ/DDQVFIHQFIDV5 HWD ,QYHWWपा प( [SШGG FUFDVvol. 5, p. 196-204.

TOMASI, R. A. (2018). "El uso de cuestionarios virtuales en plataforma Moodle previo a exámenes parciales escritos

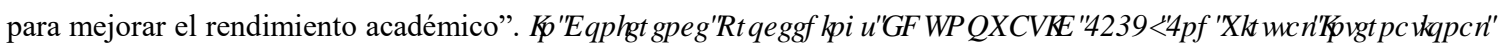
\&RQHHQFHRQ( GXFDURQI, QQRYDIRQDQG, \&7, p. 165.

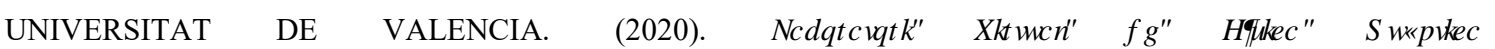
$<$ https:/www.uv.es/uvweb/departamento-fisica-teorica/es/laboratorio-virtual-fisica-cuantica-1286086076802.html> [consulta 15.02.2021].

UNIVERSIDAD EUROPEA. (2020). MyLabs - Laboratorios virtuales < https://universidadeuropea.es/experienciauniversitaria/servicios/mylabs-laboratorios-virtuales> [consulta 15.02.2021].

UNIVERSITAT INTERNACIONAL DE CATALUNYA. (2020). / DERLURUR YILWD $<$ http://www.uic.es/es/salud/laboratorios/laboratorio-virtual $>$ [consulta 15.02.2021].

UNIVERSIDAD POLITECNICA DE MADRID. (2020). / DERURRURV 9ILWDOAV 830 口 $\square$ https://3dlabs.upm.es/index.php> [consulta 15.02.2021].

VARY, J.P. (2000). "Informe de la reunión de expertos sobre laboratorios virtuales, organizada por el Instituto Internacional de Física Teórica y Aplicada (IITAP) ”, Ames, Iowa, con el apoyo de la UNESCO. París.

VINCENT-LANCRIN, S y KÄRKKÄINEN, K. (2013). “Sparking Innovation in STEM Education with Technology and Collaboration". OECD Educ. Work. Pap. 91.

ZULUAGA TORO, C.A., SÁNCHEZ TORO, C.G., RODRÍGUEZ ORTIZ, E.A. (2010). "Laboratorio de Automática Vía Internet (LAVI) ". 5HШ, EHRDP $\$$ XIRP i UFDH, QRLP $i$ UFD, QG vol. 2, p. 30-35. 